\title{
A balancing act: Physical balance, through arousal, influences size perception
}

\author{
Michael N. Geuss and Jeanine K. Stefanucci \\ University of Utah, Salt Lake City, Utah \\ AND \\ Justin de Benedictis-Kessner and Nicholas R. Stevens \\ College of William and Mary, Williamsburg, Virginia
}

\begin{abstract}
Previous research has demonstrated that manipulating vision influences balance. Here, we question whether manipulating balance can influence vision and how it may influence vision-specifically, the perception of width. In Experiment 1, participants estimated the width of beams while balanced and unbalanced. When unbalanced, participants judged the widths to be smaller. One possible explanation is that unbalanced participants did not view the stimulus as long as when balanced because they were focused on remaining balanced. In Experiment 2, we tested this notion by limiting viewing time. Experiment 2 replicated the findings of Experiment 1, but viewing time had no effect on width judgments. In Experiment 3, participants' level of arousal was manipulated, because the balancing task likely produced arousal. While jogging, participants judged the beams to be smaller. In Experiment 4, participants completed another arousing task (counting backward by sevens) that did not involve movement. Again, participants judged the beams to be smaller when aroused. Experiment $5 \mathrm{~A}$ raised participants' level of arousal before estimating the board widths (to control for potential dual-task effects) and showed that heightened arousal still influenced perceived width of the boards. Collectively, heightened levels of arousal, caused by multiple manipulations (including balance), influenced perceived width.
\end{abstract}

Postural control refers to the control of the body's position in space for the purposes of balance and orientation (Horak, 1987; Woollacott \& Shumway-Cook, 2002). Many manipulations of visual stimuli have been shown to influence the control of posture, or the variation in deviations from the center of pressure. Manipulations of illumination levels (Edwards, 1946; Paulus, Straube, \& Brandt, 1984), spatial frequency of a visual stimulus (Kunkel, Freudenthaler, Steinhoff, Baudewig, \& Paulus, 1998), motion parallax (Bronstein \& Buckwell, 1997), optical flow (Andersen \& Dyre, 1989; Bardy, Warren, \& Kay, 1999; Bertenthal, Rose, \& Bai, 1997; Dijkstra, Schoner, Giese, \& Gielen, 1994; D. N. Lee \& Aronson, 1974; D. N. Lee \& Lishman, 1975; Stoffregen, 1985), and the distance to a viewed object (Kapoula \& Thanh-Thuan, 2006; D. N. Lee \& Lishman, 1975; Stoffregen, Smart, Bardy, \& Pagulayan, 1999) can all induce greater variations in postural sway.

Although there is a large amount of literature that discusses specific influences of vision on postural sway, few studies have investigated the influence of postural sway on visual perception. The purpose of the present study was to determine whether greater postural sway (or physical states of imbalance) may also influence the perception of visual stimuli. In addition, we investigated mechanisms by which postural sway could influence perception (e.g., level of arousal). To motivate the present experiments, we will first review the literature that suggests that visual information influences postural sway. Then, we will review the scant literature that suggests that postural sway may also influence visual perception. Finally, we will discuss how the proposed experiments will contribute to this body of work.

\section{Vision Alters Postural Sway}

The earliest and clearest connection between vision and posture was found by simply asking people to close their eyes. In these studies, researchers found that closing the eyes or reducing illumination in the environment increased postural sway (Edwards, 1946; Paulus et al., 1984). Furthermore, the amount of sway induced by reduced illumination increased with age (Lord \& Ward, 1994). In addition to variations in the illumination in the environment, researchers found that the structure of the environment-specifically, the frequency of vertical lines on a flat surface - also influenced postural sway (Kunkel et al., 1998). The researchers varied spatial frequency and measured the resulting sway. They found that participants were most stable when viewing medium spatial frequencies $(2 / 3,4 / 3$ cycles per degree, in their study). This research provides one example of how static visual scenes can influence postural sway.

Researchers have also studied the relationship between postural sway and vision using the "moving room" para-

M. N. Geuss, michaelgeuss@gmail.com 
digm, in which the walls and ceilings are moved about a person in order to induce a feeling of self-motion (Andersen \& Dyre, 1989; Bardy et al., 1999; Bertenthal et al., 1997; Bronstein \& Buckwell, 1997; Dijkstra et al., 1994; D. N. Lee \& Aronson, 1974; D. N. Lee \& Lishman, 1975; Stoffregen, 1985). Specifically, optic flow generated by the room moving around the individual is attributed not to the movement of the room but to a feeling of motion in the observer. In this paradigm, experimenters generally observe that movement of the room around the participant in one direction induces a feeling of movement of the individual in the opposite direction. In response to this feeling of movement, participants often sway in the direction that the room is moving.

This relationship was first reported when D. N. Lee and Aronson (1974) noticed that children who were beginning to stand would sway when the room moved around them. Researchers also observed this effect in the trunk movements of infants as young as 5 months old who could sit independently (Bertenthal et al., 1997; Butterworth \& Hicks, 1977). Bertenthal et al. studied the effects of frequency and amplitude of optical flow (the speed of movement of the room) on postural sway of sitting infants, $5,7,9$, and 13 months of age. They concluded that children were able to visually modulate their postural responses before being able to maintain postural equilibrium. In other words, a link between the visual environment (optic flow) and balance existed even before children had the muscle capability to maintain a sitting posture.

Furthermore, research has shown that this close relationship between vision and postural control persists in adults. Specifically, researchers have found that restricting vision of the moving room to the periphery of the visual field was sufficient to induce postural sway (Bertenthal \& Bai, 1989; Stoffregen, Schmuckler, \& Gibson, 1987). This finding suggests that with restricted vision, alterations in optic flow detected in the periphery of the visual field are sufficient to induce postural fluctuations. For the purposes of the present experiments, this series of research provides a clear example that alterations to the visual environment produce changes in postural sway.

An oft-raised criticism of the moving room paradigm is that participants have to remain completely still for the visual flow generated by the moving room to induce sway. However, postural control is nonetheless important because it can facilitate the achievement of a nonpostural task, or a task where the primary goal is not just maintaining a stable posture (Riccio \& Stoffregen, 1988; Stoffregen et al., 1999). In this manner, increased variations in postural sway may be adaptive for the completion of a suprapostural task. In a series of experiments, Stoffregen et al. (1999) instructed participants to fixate on a nearby or distant target and recorded the resulting amount of lateral and sagittal sway. Their task was to "stare intently" at a near object, a far object with a nearby object present in the visual field, or a far object without a nearby object present. Stroffregen et al. (1999) found that participants' variation in sway was greater when they were focusing on the far object than when they were focusing on the near object. However, there were no differences due to the presence or absence of an object nearby when they were fixating on the far object. The authors concluded that there were no differences between focusing on the far object with or without a nearby object, because the nearby object was irrelevant for completing the suprapostural task. This experiment exemplifies not only the importance of the visual environment, but also the effect that the requirements of the suprapostural task can have on the amount of postural sway.

Collectively, these studies demonstrate the importance of visual information to the maintenance of balance. Furthermore, visual tasks may benefit from different amounts of postural sway, and these differences in postural sway may not always be dangerous (e.g., increase the risk of falls; Stoffregen et al., 1999). However, little research has been done to test the influence of postural sway on perceiving the visual environment. It seems likely that if visual stimuli largely affect postural sway, variations in sway may also change the processing of visual information.

\section{Postural Sway Influences Vision}

Several lines of research suggest that postural sway can influence higher level cognitive processes. This research has found that greater postural sway negatively influenced performance on a spatial memory task (Kerr, Condon, \& McDonald, 1985) and reduced reaction times for an auditory stimulus (Lajoie, Teasdale, Bard, \& Fleury, 1993). Kerr et al. investigated the influence of postural sway on participants' performance on a spatial memory task. The researchers manipulated balance by instructing participants to stand in the Romberg position, where the heel of one foot was directly in front of the toes of the other. Participants completed either a spatial memory task (remembering numbers and their relative locations) or a nonspatial memory task (remembering only numbers, without an associated location) both while in the Romberg position and while not. The authors found that only the spatial memory task was negatively influenced by the balance task. Performance was no different between stable and unstable postures on the nonspatial memory task. The authors concluded that spatial memory was negatively affected by the balance task because the spatial memory and balance tasks shared resources devoted to visual processing.

Postural sway has also been shown to influence attention (Lajoie et al., 1993). Lajoie et al. varied the postural stability of participants by instructing them to sit or stand and measured reaction times to an auditory stimulus. They found that greater postural sway, induced when standing, influenced reaction times, such that participants were faster to react when sitting stably than when standing upright. Collectively, these results suggest that postural sway influences cognitive functioning and may also influence a lower level process such as visual perception.

Furthermore, researchers have also investigated the influence that restraints on movement have on learning new affordances, or what one is capable of doing given the constraints of the environment and the body (Mark, Balliet, Graver, Douglas, \& Fox, 1990). Mark et al. studied the 
influence of postural sway on visual perception; specifically, they suggested that postural motion can be helpful in acquiring information about changes in a person's action capabilities. To test this hypothesis, the authors altered the length of participants' legs by strapping $10-\mathrm{cm}$ extensions to the soles of their feet. These extensions rescaled action decisions that normally reference leg height as information for the decision. For example, when deciding whether a seat is too high to sit on, individuals must reference the height of their legs. Extending height makes one able to sit on objects that were once too tall. Once participants donned the leg extensions, they were instructed either to lean against a wall or to stand normally. Participants who leaned against the wall were restricted from experiencing sway normally associated with standing. Mark et al. found that when sway was restricted, the participants did not learn to incorporate their longer legs into their action decisions. In other words, participants reported that they could not sit on taller heights when they actually could, due to their extended height. Two nonexclusive explanations exist to explain this finding: (1) Postural sway allowed participants to better perceive their new height and thus perceive the affordance, and (2) greater amounts of postural sway created a richer visual experience (e.g., optic flow, motion parallax). In this experiment, postural sway allowed participants to more accurately judge the actions that they were capable of performing.

If greater postural sway affects other cognitive processes, such as spatial memory and attention, it is possible that it also affects visual perception. Mark et al. (1990) confirmed that postural sway influenced one measure of visual perception: the perception of affordances. However, the perception of affordances is likely related to an individual's perception of the size or extent of the environmental object being judged. In the case of Mark et al.'s findings, one question to ask is whether the leg extensions and/or postural constraints influenced participants to see the seating structure as being a different height, before or when judging their ability to sit on it. Here, we question whether postural sway may also influence the perceived size of objects in the environment.

\section{Overview of Present Experiments}

In the present study, we investigated the influence of postural sway on the visual perception of width. To our knowledge, we are the first to investigate the influence of postural sway on the perception of spatial properties. In Experiment 1, we asked participants to estimate the width of a beam that they were told to imagine walking across. Participants judged the width while performing a balance task. In Experiments 2, 3, 4, and 5, we attempted to determine whether two underlying mechanisms, attention and arousal, contributed to the results of Experiment 1. Thus, in Experiment 2, we tested whether, when unbalanced, participants may have also diverted their attention from the beam. Then we conducted experiments to determine whether experiencing heightened levels of arousal when imbalanced (Experiment 3 ) and gross head movements (Experiment 4) contribute to an effect of balance on perception. The results of Experiments 2-4 suggested that arousal, rather than attention, was a likely cause of our original findings in Experiment 1. Therefore, Experiments $5 \mathrm{~A}$ and $5 \mathrm{~B}$ tested the influence of heightened arousal, without head movements or concurrent tasks, on estimates of size perception.

\section{EXPERIMENT 1}

In this experiment, we manipulated participants' balance while they estimated the width of beams. Proffitt (2006) stated that perception of the environment is moderated by the costs of acting in the environment. In this experiment, a state of physical imbalance may serve to increase the perceived costs of acting and then adaptively change the perceived beam width. Therefore, we hypothesized that, when unbalanced, participants would estimate the width of the beam to be smaller than when they were balanced.

\section{Method}

Participants. Twenty ( 8 female, 12 male) College of William and Mary students participated in the experiment for credit in an introductory psychology course. All participants could maintain a normal posture and had normal or corrected-to-normal vision. All participants were naive as to the purpose of the experiment and gave written, informed consent to participate.

Apparatus. Participants judged beam widths in a $3.05 \times 3.05 \mathrm{~m}$ room with a solid colored carpet. Four beams of the same length $(121.92 \mathrm{~cm})$ but different widths $(1.75,3.65,8.73$, or $13.5 \mathrm{~cm})$ were placed on top of two vertically standing cinder blocks $(19 \mathrm{~cm}$ wide, $39.37 \mathrm{~cm}$ tall). The beams were placed in front of the participants such that the length of the beam extended directly in front of the location where they stood (home position). The closest part of the beam was $30.58 \mathrm{~cm}$ from the home position (see Figure 1).

Participants stood on top of a balance board (GoFit 15-in. round wood balance board). The balance board was $39.37 \mathrm{~cm}$ in diameter and $6.35 \mathrm{~cm}$ high when horizontal. In the balanced condition, the

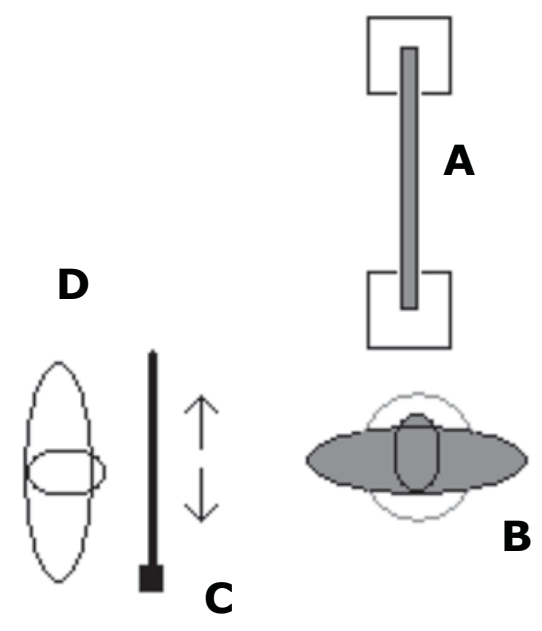

Figure 1. View of the experimental setup for all experiments. The participant $(B)$ stood atop the balance board and judged the width of the beam (A). For the visually matched estimates, the experimenter (D) pulled out the tape measure (C) to match the width of the beam. 


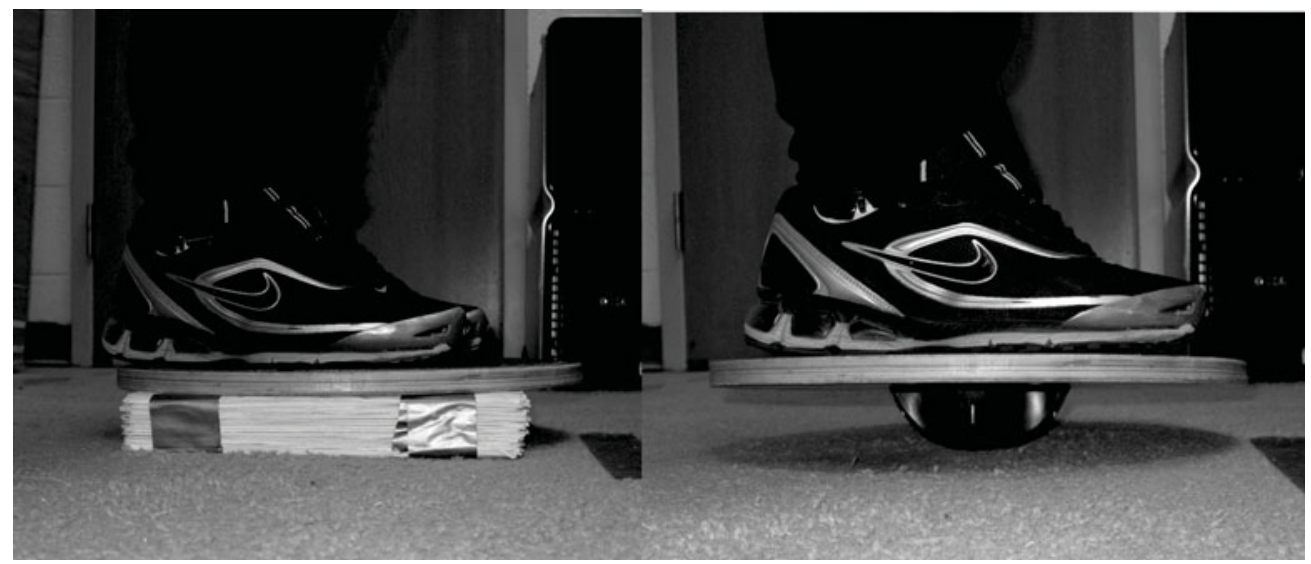

Figure 2. Participants judged the width of the beam while both balanced (left) and unbalanced (right). In the unbalanced condition, the balance board rotated about its center point. The participant was instructed to keep the board horizontal.

board was stabilized by placing two blocks underneath the board (see Figure 2A). In the unbalanced condition, the balance board rotated around a center point (see Figure 2B). A standard, 365.76-cm tape measure was used for the visual matching task.

Design. A within-participants design was used; therefore, all participants were in the balanced and unbalanced conditions. Half of the participants saw all the beams while balanced first, and the other half saw all the beams while unbalanced first. Additionally, for each condition (balanced and unbalanced), the participants viewed two blocks of beam widths. Each block consisted of a single presentation of each of the beam widths. The result was 4 estimates per block and two blocks per condition, for a total of 16 estimates per participant. Only 16 estimates were obtained, to ensure that participants did not become exhausted and unable to attempt to keep the board horizontal in the unbalanced condition.

Procedure. Participants stood on top of the balance board for both conditions. They were instructed to imagine walking across the presented beam and then completed a visual matching task. Participants imagined walking across the beam to evoke an intention to interact with the environment. Previous work has shown that an intention to act is necessary to rescale the perceived size of the judged extent (Witt, Proffitt, \& Epstein, 2005). To complete the visual matching task, participants were instructed to match the width of the board to the length of a tape measure. The experimenter touched the outside edges of the beam where the width was to be estimated when explaining the task and adjusted the tape measure while standing $90^{\circ}$ to the left of the participants. The experimenter always started the matching task with the tape measure outstretched and then slowly decreased its length until the participants indicated that the experimenter should stop. Participants were instructed to adjust the length of the tape measure until they felt it was of a length equivalent to the beam's width. They were told to look back and forth between the beam and tape measure as many times as they needed to be accurate with their estimates. The visual matching task was the same for both the balanced and unbalanced conditions.

Between each estimate, participants were instructed to close their eyes and grasp a nearby table to prevent falls when unbalanced. Once the beam was in place, the participants viewed the beam for an unrestricted amount of time while making their width estimate.

Once participants had completed one condition (either balanced or unbalanced), the stabilizing blocks were either removed from or added to the underside of the balance board. They were given about a minute to familiarize themselves with the balance board prior to the unbalanced condition. Throughout the unbalanced condition, participants were instructed to attempt to keep the balance board horizontal. However, keeping the board horizontal is a difficult task that required participants to constantly monitor their movement and react to deviations. When standing on the board, participants continually experienced slight to complete (edge of board touching floor) leans and sways in all directions (given that the board was round), mimicking a highly imbalanced situation. Participants were monitored and reminded to balance the board until the completion of the matching task. The entire procedure lasted about $15 \mathrm{~min}$.

\section{Results and Discussion}

Width estimates. A 2 (order: balanced or unbalanced first) $\times 2$ (condition: balanced, unbalanced) $\times 2$ (block: first, second) $\times 4$ (width: $1.75,3.65,8.73,13.5 \mathrm{~cm}) \mathrm{re}-$ peated measures ANOVA with order as the only betweenparticipants variable was run to determine the effect of balance on size perception. The analyses revealed a main effect of condition $\left[F(1,18)=4.67, M S_{\mathrm{e}}=2.39, p=.044\right.$, $\left.\eta_{\mathrm{p}}^{2}=.21\right]$. Participants estimated that the beam was significantly smaller when unbalanced $(M=8.23, S E=0.08)$

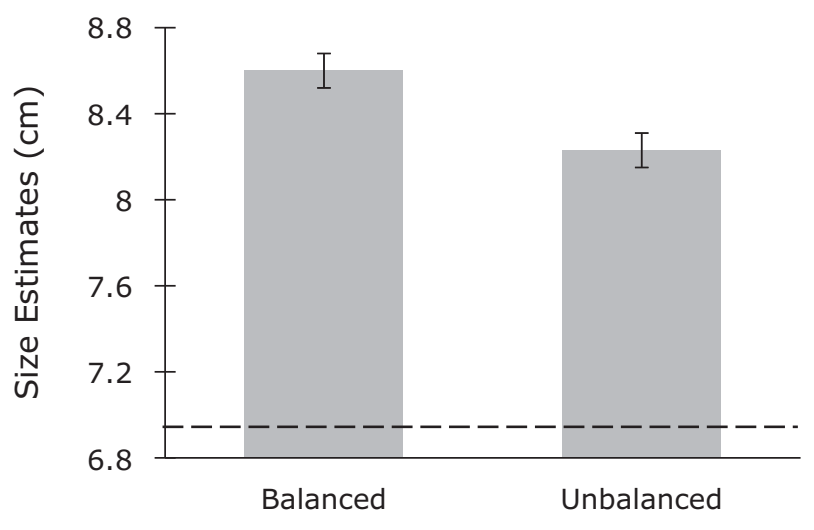

Balance Condition

Figure 3. Results for Experiment 1. The $y$-axis represents the average width estimates for all four beam widths for each condition (balanced and unbalanced). Participants judged the beam to be significantly smaller when unbalanced than when balanced. All bars represent 1 standard error. The horizontal line represents the average of the actual board widths. 
than when balanced $\left(M=8.60, S E=0.08\right.$; see Figure 3). ${ }^{1}$ There was no effect of order $[F(1,18)=0.16, p=.69]$ or block $[F(1,18)=0.07, p=.79]$ and no condition $\times$ order interaction $[F(1,18)=0.001, p=.98]$. As was expected, the analysis revealed a main effect of beam width $\left[F(3,54)=634.61, M S_{\mathrm{e}}=4.58, p<.0001, \eta_{\mathrm{p}}^{2}=.97\right]$.

Precision of estimates. We also tested whether participants' precision, or variability in error, for estimating the width of the board was influenced by condition. For example, when in the unbalanced condition, participants' attention could have been focused on the board rather than on the size estimate. This might have produced greater variability in the estimates of board width. To test whether condition influenced precision of estimates, we computed an error term for each data point.

The error term was calculated by creating an $S D$ for each data point from the mean of the two width estimates within each condition. This was repeated for both conditions, which were within participants. A repeated measures ANOVA (same as that used above) was run to test for differences in precision. There was no significant effect of condition $[F(1,18)=0.24, p=.63]$, block $[F(1,18)=$ $2.84, p=.11]$, or order $[F(1,18)=0.29, p=.60]$. There was a significant effect of width $\left[F(3,54)=3.68, M S_{\mathrm{e}}=\right.$ $\left.153.37, p=.02, \eta_{\mathrm{p}}^{2}=.17\right]$. Participants' estimates of width were more precise when estimating smaller board widths. The null result of condition suggests that balance condition did not influence variability in participants' estimates of board widths.

The results indicated that physical imbalance affected participants' estimates of beam widths when they imagined walking across the beam. Specifically, when participants were unbalanced, they reported perceiving the beam's width as smaller than when balanced. However, it is not clear which aspect of physical imbalance contributed to this effect. An alternative explanation is that when participants were unbalanced, they spent less time viewing the beam's width and more time viewing the balance board to sustain balance. In the next experiment, we investigated the effects of viewing time on the perception of beam widths.

\section{EXPERIMENT 2}

Experiment 2 investigated the influence of attention, manipulated by limiting viewing time, on the perceived width of beams. Participants estimated the beam widths with an unlimited viewing time and a limited viewing time. For each viewing-time condition, participants judged widths while balanced and unbalanced. We hypothesized that if attention contributed to the results of Experiment 1, then when the viewing time was limited, participants would judge the width of the beam to be thinner than when allowed unlimited time.

\section{Method}

Participants. Eighteen (12 female, 6 male) College of William and Mary students participated in the experiment for credit in an introductory psychology course. All participants could maintain a normal posture and had normal or corrected-to-normal vision. All participants were naive as to the purpose of the experiment and gave written informed consent to participate.

Apparatus. All apparati were the same as in Experiment 1.

Design. A within-participants design was used; therefore, all participants completed both viewing-time (limited and unlimited) and balance (balanced and unbalanced) conditions. The order of viewing-time and balance conditions was randomized and counterbalanced. Half of the participants viewed the beams with an unlimited viewing time first, and the other half with a limited viewing time first. Within each viewing time, half of the participants saw the beams either when balanced or when unbalanced first. The order of balance trials was consistent across viewing times. Specifically, if, during the first viewing condition, participants made judgments while balanced first, then in the second viewing condition, they also made judgments while balanced first.

Within each viewing-time condition, the participants judged each beam eight times (once per block) for each of the two balance conditions. Therefore, each participant made 32 judgments, 16 for each of the viewing-time conditions and 16 for each of the balance conditions.

Procedure. All procedures were the same as in Experiment 1, except that during the limited viewing time, participants saw the beam for only $3 \mathrm{sec}$. After $3 \mathrm{sec}$, the experimenter removed the beam and placed it out of sight. The participant closed his or her eyes while the board was being removed, which usually took no longer than $5 \mathrm{sec}$. The participant then completed the visual matching task in the same manner as that described in Experiment 1; however, they were not able to reference the beam as they estimated its width because it was out of sight. The entire procedure lasted about $30 \mathrm{~min}$.

\section{Results and Discussion}

Width estimates. A 2 (order: first, second) $\times 2$ (viewing time: limited, unlimited) $\times 2$ (condition: balanced, unbalanced) $\times 2$ (block: first, second) $\times 4$ (width: 1.75 , $3.65,8.73,13.5 \mathrm{~cm})$ repeated measures ANOVA, with order as the only between-participants factor, revealed a significant main effect of condition $[F(1,14)=6.19$, $\left.M S_{\mathrm{e}}=1.85, p=.03, \eta_{\mathrm{p}}^{2}=.31\right]$. When unbalanced, participants judged the beam to be significantly smaller $(M=7.64, S E=0.05)$ than when balanced $(M=7.97$, $S E=0.05)$. There was also a significant effect of block $\left[F(1,14)=9.59, M S_{\mathrm{e}}=1.00, p=.01, \eta_{\mathrm{p}}^{2}=.41\right]$. Participants judged the beams to be significantly larger in the first block $(M=7.95, S E=0.21)$ than they did in the second block $(M=7.66, S E=0.19)$. There was no significant effect of viewing time $[F(1,14)=1.11, p=.31]$ or order $[F(3,14)=0.05, p=.99]$. There was no interaction between viewing time and balance $[F(1,14)=1.34, p=$ $.26]$ or between condition and order $[F(3,14)=0.13, p=$ .94]. There was also no interaction between condition and block $[F(1,14)=0.25, p=.62]$ or between time and block $[F(1,14)=0.62, p=.44]$. As was expected, there was a main effect of width $\left[F(3,42)=461.98, M S_{\mathrm{e}}=8.02, p<\right.$ $\left..0001, \eta_{\mathrm{p}}^{2}=.97\right]$.

As in Experiment 1, participants reported perceiving the beam as smaller when unbalanced than when balanced. However, results also indicated that regardless of balance condition, viewing time did not influence their estimates of beam widths. The results also revealed a main effect of block. Regardless of condition and viewing time, participants estimated the beam widths as thinner the second time they judged them. This finding could be the result of practice effects; however, it is difficult to interpret, 
given that there was no interaction of block with time or condition. Collectively, the results of this experiment suggest that the effect of balance found in Experiment 1 was probably not due to attention.

In the following experiment, we tested whether an increase in arousal, when produced during imbalance and jogging, results in a change in size perception. Given that the balance board is a commercially sold product designed for exercise purposes, we thought that increases in the level of arousal associated with being imbalanced or exercising could underlie changes in perception.

\section{EXPERIMENT 3}

In this experiment, we manipulated the participants' balance and level of arousal while they judged the width of a beam. Participants jogged in place to raise their level of arousal. We believe that because the balance board is an exercise tool, participants may have also experienced higher rates of arousal when unbalanced in the previous experiments. Previous work suggests that arousal can influence the perception of spatial layout (Stefanucci \& Storbeck, 2009). Specifically, Stefanucci and Storbeck found that arousal increased perceived height. Here, we tested whether arousal can influence perceived size. We hypothesized that participants would estimate the width of the beam to be smaller when they were unbalanced and when jogging than when they were balanced.

\section{Method}

Participants. Twelve ( 3 female, 9 male) College of William and Mary students participated in the experiment for credit in an introductory psychology course. All participants could maintain a normal posture and had normal or corrected-to-normal vision. All participants were naive as to the purpose of the experiment and gave written informed consent to participate.

Apparatus. All apparati were the same as those used in Experiments 1 and 2 .

Design. A within-participant design was used; therefore, all participants completed all three conditions (balanced, unbalanced, and jogging). The order of the conditions was randomized and counterbalanced. Within each condition, the participants viewed each beam twice. The four beam widths were blocked and randomly presented within each block. The result was eight estimates per condition, for a total of 24 beam judgments per participant.

Procedure. All procedures were the same as in Experiment 1, except that participants also jogged in place while estimating the beam widths. Participants were instructed to begin jogging when they opened their eyes and continued to jog until they had completed their estimates. Experimenters monitored the rate of jogging so that participants did not slow, thereby keeping their arousal level high. Participants were also given a short break between conditions to minimize the arousal of one condition affecting arousal in the subsequent conditions. The entire procedure lasted no more than $20 \mathrm{~min}$.

\section{Results and Discussion}

Width estimates. A 3 (order) $\times 3$ (condition: balance, unbalanced, jogging) $\times 2$ (block: first, second) $\times$ 4 (width: $1.75,3.65,8.73,13.5 \mathrm{~cm}$ ) repeated measures ANOVA, with order as the only between-participants factor, revealed a main effect of condition $[F(2,18)=3.69$, $\left.M S_{\mathrm{e}}=3.68, p=.045, \eta_{\mathrm{p}}^{2}=.29\right]$. As was expected, there was also a main effect of width $\left[F(3,27)=330.24, M S_{\mathrm{t}}=\right.$

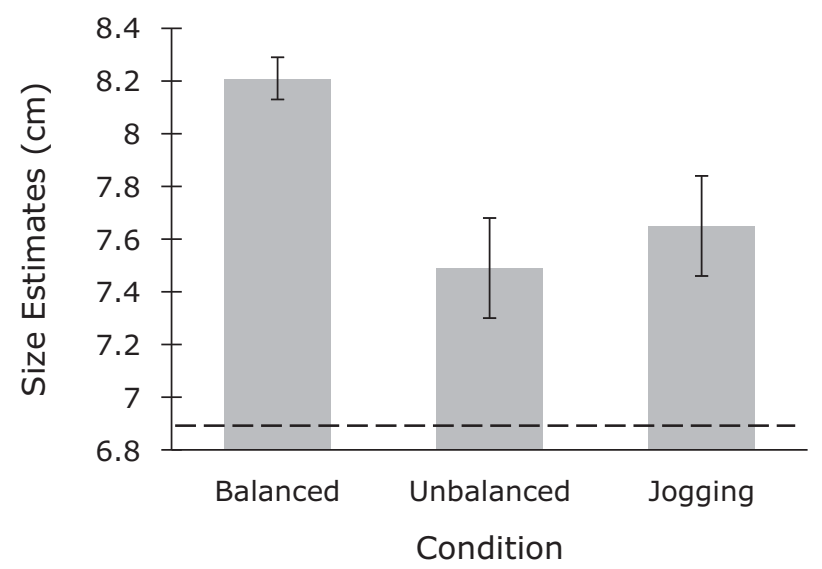

Figure 4. Results for Experiment 3. The $y$-axis represents the average width estimate for each of the three conditions (balanced, unbalanced, and jogging). Participants judged the width of the beam to be significantly smaller when in the jogging and unbalanced conditions than when in the balanced condition. The jogging and unbalanced conditions did not differ from one another. All bars represent 1 standard error. The horizontal line represents the average of the actual board widths.

$\left.7.80, p<.0001, \eta_{\mathrm{p}}^{2}=.97\right]$. However, there was no effect of order $[F(2,9)=0.71, p=.52]$ or block $[F(1,9)=0.45$, $p=.52]$ and no interaction between order and condition $[F(4,18)=0.11, p=.98]$.

Post hoc analysis using Fisher's LSD revealed significant differences between the balance condition and the unbalanced condition $(p=.009)$. Participants estimated the beam widths to be significantly smaller when unbalanced $(M=7.49, S E=0.19)$ than when balanced $(M=$ $8.21, S E=0.08)$. Additionally, participants judged the beam to be significantly smaller when jogging $(M=$ 7.65, $S E=0.19)$ than when balanced $(M=8.21, S E=$ $0.08 ; p=.04)$. However, no differences between the jogging and unbalanced conditions were found $(p=.67$; see Figure 4).

Precision of estimates. From a motivation similar to that in Experiment 1, we analyzed whether jogging influenced the precision with which participants estimated the boards' widths. Error terms were created as in Experiment 1 . The same repeated measures ANOVA used to evaluate changes in mean estimations of width was used to evaluate the error terms. There was no significant effect of condition $[F(2,18)=0.95, p=.41]$, block $[F(1,9)=$ $1.34, p=.27]$, or order $[F(2,9)=0.78, p=.49]$. There was a significant effect of width $\left[F(3,27)=8.12, M S_{\mathrm{e}}=\right.$ $\left.49.89, p=.001, \eta_{\mathrm{p}}^{2}=.47\right]$. Participants' estimates of width were more precise when estimating smaller board widths. The null result of condition suggests that an increase in arousal, likely produced by jogging, did not influence the variability of estimates.

Again, the results indicated that being unbalanced affected the perception of width. Furthermore, the results indicated that another arousing task, jogging, affected the perception of beam width as well. Collectively, the results suggest that a higher state of arousal when unbalanced may contribute to the results originally found in 
Experiment 1. However, both the unbalanced and jogging conditions also involved more gross movements of the body than did the balanced condition. In the next experiment, we tested whether an arousing task that did not also require gross movements was sufficient to produce the changes in perception found in the previous three experiments.

\section{EXPERIMENT 4}

In this experiment, we manipulated arousal without having participants concurrently make large movements. Specifically, participants judged the width of beams when counting backward by sevens (counting) and without counting (no counting). Participants were balanced in both conditions. The counting task has reliably and consistently been shown to raise physiological arousal (Budzynski \& Peffer, 1980; Harvey, 1980; S. W. Lee \& Guck, 1990; Maki \& McIlroy, 1996; Noteboom, Fleshner, \& Enoka, 2001; Schneider, 1979; Yardley, Watson, Britton, Lear, \& Bird, 1995). We hypothesized that even without gross body movements, when participants were more aroused due to counting, they would estimate the beam widths to be smaller than when balanced and not aroused.

\section{Method}

Participants. Fifteen (10 female, 5 male) College of William and Mary students participated in the experiment for credit in an introductory psychology course. All participants could maintain a normal posture and had normal or corrected-to-normal vision. All participants were naive as to the purpose of the experiment and gave written informed consent to participate.

Apparatus. All apparati were the same as in Experiment 1, except that the size-matching task was completed using the computer screen of a Dell Inspiron 1521 laptop running MATLAB. Participants were able to press up and down arrow keys to position two vertical lines on the laptop screen to be the same width as the beam.

Design. A within-participants design was used; therefore, all participants completed width estimates in the nonarousing condition (no counting) and the arousing condition (counting). The order of conditions was randomized and counterbalanced. Within each condition, participants judged each of the four beam widths twice, for a total of 16 estimates.

Procedure. All procedures were the same as in Experiment 1, except that the unbalanced condition from Experiment 1 was replaced with a counting condition. In the counting task, participants were given a random three-digit number at the beginning of each trial. They continually counted out loud backward by sevens until they had completed the size-matching task. Participants were instructed to complete the subtractions quickly and were encouraged to give a response if they slowed their responses. A second experimenter recorded the numbers that participants verbalized.

In previous experiments, participants verbally adjusted the tape measure. In the present experiment, it would have been difficult for participants to complete the counting task and verbally adjust the tape measure. Therefore, the size-matching task utilized a MATLAB program that initially displayed two vertical lines at a random distance from each other. In this manner, participants completed both ascending and descending trials. Participants were instructed to push the up and down arrows on the laptop keyboard to adjust the distance between the two vertical lines to be equal to the width of the beams. The laptop version of the visually matched estimates allowed participants to complete the matching task in a way that did not interfere with counting backward. The entire procedure lasted about $20 \mathrm{~min}$.

\section{Results and Discussion}

Counting task. The numbers were scored such that any number that was not 7 less than the previous number was scored as an error. The total number of errors was divided by the total number of subtractions spoken for each participant. This standardized the percentage of error across participants. All participants responded correctly at least $74 \%$ of the time, and all were included in the analysis.

Width estimates. A 2 (order) $\times 2$ (condition: no counting, counting) $\times 2$ (block: first, second) $\times 4$ (width: $1.75,3.65,8.73,13.5 \mathrm{~cm})$ repeated measures ANOVA, with order as the only between-participants factor, revealed a main effect of condition $\left[F(1,13)=6.71, M S_{\mathrm{e}}=\right.$ $\left.1.21, p=.02, \eta_{\mathrm{p}}^{2}=.34\right]$. Participants judged the beams to be significantly smaller when counting $(M=6.75, S E=$ $0.07)$ than when not counting $(M=7.12, S E=0.07$; see Figure 5). There was no effect of order $[F(1,13)=0.45$, $p=.52]$ and no interaction between condition and order $[F(1,13)=1.17, p=.30]$. There was also no effect of block $[F(1,13)=1.11, p=.31]$. In addition, there was a main effect of beam width $\left[F(3,42)=377.25, M S_{\mathrm{e}}=\right.$ $\left.4.11, p<.0001, \eta_{\mathrm{p}}^{2}=.96\right]$.

Precision of estimates. From a motivation similar to that in Experiments 1 and 3, we analyzed whether counting influenced the precision with which participants estimated the board widths. Error terms were created as in Experiments 1 and 3 . The same repeated measures ANOVA analysis used to evaluate changes in mean estimations of width was used to evaluate the error terms. There was no significant effect of condition $[F(1,13)=0.003, p=.96]$, block $[F(1,13)=1.25, p=.28]$, or order $[F(2,9)=0.78, p=$ .49]. There was a significant effect of width $[F(3,39)=$ 29.14, $\left.M S_{\mathrm{e}}=15.6, p<.001, \eta_{\mathrm{p}}^{2}=.69\right]$. As in Experiments 1 and 3, participants' estimates were more precise when estimating smaller board widths. The null result of condition suggests that increased arousal, which likely occurred in the counting task, did not influence precision.

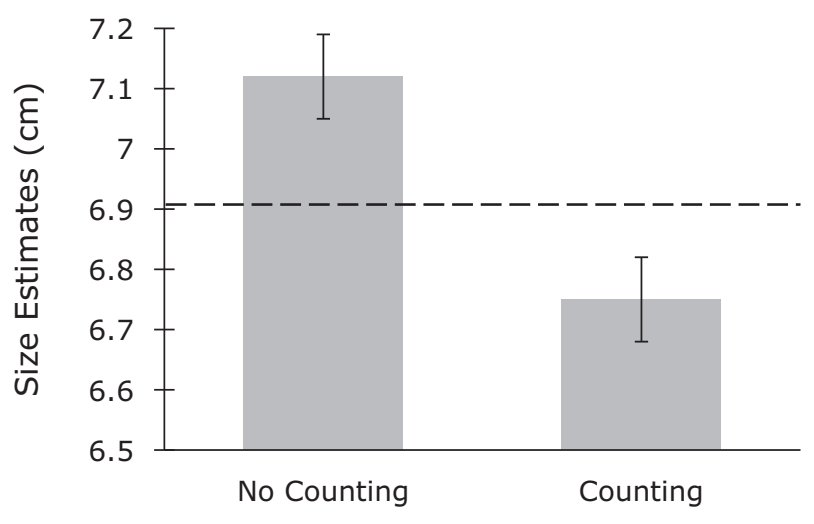

Arousal Condition

Figure 5. Results for Experiment 4. The $y$-axis represents the average width estimates for the no-counting and counting conditions. Participants judged the width to be significantly smaller when performing the counting task. All bars represent 1 standard error. The horizontal line represents the average of the actual board widths. 
The results indicated that higher levels of arousal in the counting condition were sufficient to alter the perception of environmental layout when participants had imagined walking across the beam. Furthermore, the results suggested that gross body movement (as seen in the unbalanced condition of Experiments 1 and 2 and the jogging condition of Experiment 3) was not necessary to produce the effects. We cannot make any conclusions about the influence of movement alone (without arousal) on perception, but the results suggest that higher levels of arousal, as produced when one is unbalanced, jogging, and counting, are sufficient to affect perception.

\section{EXPERIMENT 5A}

In all the previous conditions (unbalanced, jogging, and counting) that produced an effect, participants were aroused, but also performed two tasks simultaneously. In this experiment, we manipulated level of arousal before participants estimated the width of the board. Participants viewed arousing and neutral pictures selected from the International Affective Picture System (IAPS; Lang, Bradley, \& Cuthbert, 1999). Previous studies have found that viewing the arousing pictures reliably raises physiological arousal, in comparison with viewing the neutral pictures (Bradley, Codispoti, Cuthbert, \& Lang, 2001; Bradley \& Lang, 2007). Participants were aroused without performing a secondary task while estimating the boards' widths. We expected that when participants were more aroused, they would estimate the width of the board as thinner.

\section{Method}

Participants. Eleven ( 9 female, 2 male) University of Utah students participated in the experiment for credit in a psychology course. All participants could maintain a normal posture and had normal or corrected-to-normal vision. All participants were naive as to the purpose of the experiment and gave written informed consent to participate.

Apparatus and Stimuli. All apparati were the same as those used in Experiments 1, 2, and 3, except for the addition of pictures selected from Lang et al.'s (1999) IAPS. One hundred twenty images were selected and divided into two groups (neutral and arousing) of 60 pictures. ${ }^{2}$ The arousing pictures were rated as significantly more arousing $(M=6.1, S E=0.08)$ than the neutral set of pictures $(M=$ $4.2, S E=0.09)[t(118)=14.22, p<.001]$. Valence was rated no differently between the arousing set $(M=5.4, S E=1.8)$ and the neutral $\operatorname{set}(M=5.3, S E=0.26)$ of pictures $[t(118)=0.11, p=.91]$. Images were presented using PowerPoint.

Design. A within-participants design was used. All participants completed the neutral condition followed by the arousing condition. The order of conditions was always neutral and then arousing to reduce possible carryover effects of the arousing images. If the arousing condition preceded the nonarousing condition, it is likely that participants' level of arousal would not have decreased to a baseline level of arousal for the neutral condition. For both conditions, participants judged two blocks of four beam widths. Therefore, each participant made a total of 16 estimates.

Procedure. Upon arrival, participants were led to believe that they were going to participate in two separate pilot experiments. For one of the two experiments, participants were told that they would view a series of pictures; their task was to view the pictures and to think about why each was interesting to them. Participants were told that, later in the experiment, they would see each picture again and would be asked to describe to the experimenter what they found in- teresting. Participants were not required to memorize anything about the pictures. The experimenter explained that due to the large number of pictures, there would periodically be breaks between viewings of the images. During these breaks, the participants estimated the widths of the boards, under the guise that this procedure was a separate experiment. The procedure in the second experiment was identical to that in the balanced and control conditions in Experiments 1, 2, and 3. Participants judged the same four board widths, using the visually matched estimation task with the tape measure. Participants adjusted the length of the tape measure to be equal to the width of the board.

Each participant viewed a set of neutral pictures, estimated the width of the boards, viewed a set of arousing pictures, and then estimated the width of the boards a second time. All pictures were selected from the IAPS library. Each set of pictures contained $60 \mathrm{im}-$ ages. Each picture was played on a computer screen for $3 \mathrm{sec}$. After all pictures in a set were viewed ( $3 \mathrm{~min}$ ), participants were led to another room containing the boards.

After viewing the arousing (second) set of pictures and estimating the widths of the board, participants were asked a series of questions. First, they were asked whether they could verbalize the hypothesis of the experiment. This was asked in order to identify participants who had guessed the hypothesis and may have cognitively altered their estimates. Participants then rated how aroused they felt while viewing the first and second sets of pictures. Responses were made on a scale of 1 to 7 , where a 1 was not at all arousing and a 7 was extremely arousing. They were then debriefed about the true nature of the study. The entire procedure lasted around $20 \mathrm{~min}$.

\section{Results and Discussion}

Response bias check. Due to the within-participants nature of the study, participants could have guessed the hypothesis and changed their estimates accordingly. However, none of the participants were able to verbalize the true hypothesis of the experiment. Most made statements similar to ". . . to see how accurate people are at estimating widths" or ". . . to see what I thought was interesting about the pictures." Two participants said they suspected the "two studies" might be related. However, neither participant correctly verbalized the relationship. We are confident that participants believed that they participated in two separate experiments. This leads us to believe that participants did not adjust their estimates due to some cognitive bias or hypothesis about the nature of the experiments.

Arousal ratings. A paired-samples $t$ test was used to compare participants' ratings of their level of arousal when viewing each set of pictures. Analyses revealed that participants were significantly more aroused when viewing the arousing set of pictures $(M=4.86, S E=0.42)$ than when viewing the less arousing pictures $(M=2.36$, $S E=0.36)[t(10)=-6.27, p<.001]$.

Width estimates. A 2 (condition: neutral, arousing) $\times$ 2 (block: first, second) $\times 4$ (width: $1.75,3.65,8.73,13.5 \mathrm{~cm}$ ) repeated measures ANOVA, with all factors as within participants, revealed a significant main effect of condition $\left[F(1,10)=17.25, M S_{\mathrm{e}}=.32, p=.002, \eta_{\mathrm{p}}^{2}=.63\right]$. Participants estimated the boards to be significantly thinner when in the arousing condition $(M=8.06, S E=0.11)$ than when in the neutral condition $(M=8.92, S E=0.11$; see Figure 6). There was no main effect of block $[F(1,10)=$ $2.88, p=.22]$. There was a significant main effect of width $\left[F(3,30)=584.75, M S_{\mathrm{e}}=.36, p<.001, \eta_{\mathrm{p}}^{2}=.98\right]$. 


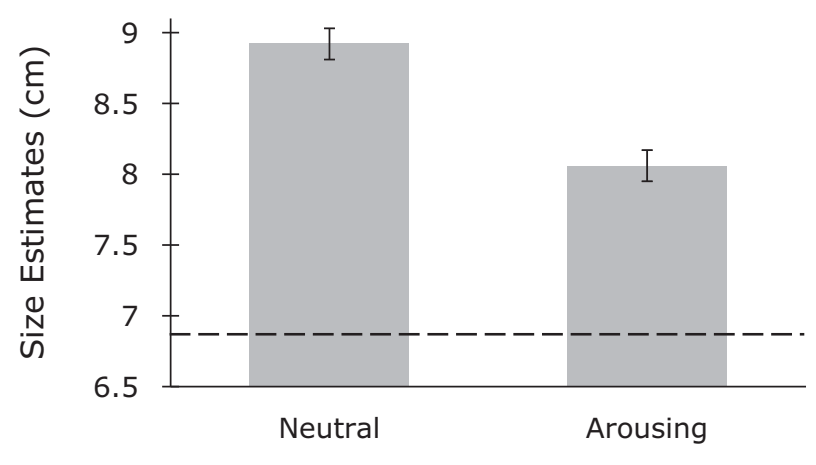

Arousal Condition

Figure 6. Results for Experiment 5A. The $y$-axis represents the average width estimates for the neutral and arousing conditions. Participants judged the width to be significantly smaller after viewing the arousing images. All bars represent 1 standard error. The horizontal line represents the average of the actual board widths.

Precision of estimates. From a motivation similar to that in Experiments 1, 3, and 4, we analyzed whether arousal influenced the precision of estimates. Error terms were created as in Experiments 1, 3, and 4. The same repeated measures ANOVA used to evaluate changes in mean estimations of width was used to evaluate the error terms. There was a trend toward significant effects of condition $[F(1,10)=4.47, p=.06]$ and block $[F(1,10)=4.8, p=$ $.053]$. The trend suggests that when aroused, participants may have been more precise $(M=.37, S E=.08)$ than when less aroused $(M=.54, S E=.13)$. Although trending, the test was nonsignificant. This, coupled with the fact that no other experiment saw a similar trend, leads us to conclude that arousal likely does not influence precision. There was a significant effect of width $[F(3,30)=4.04$, $\left.M S_{\mathrm{e}}=.55, p=.02, \eta_{\mathrm{p}}^{2}=.28\right]$. As in Experiments 1,3 , and 4, participants' estimates of width were more precise when estimating smaller board widths.

Even though they did not perform a concurrent secondary task, participants estimated the width of the board to be significantly thinner when aroused than when not aroused. The results of this experiment suggest that the change in perceived width when participants were unbalanced, jogging, and counting was due to greater levels of arousal, rather than performing dual tasks. However, participants always viewed the neutral picture set first and the arousing picture set second. Therefore, the results of this experiment could reflect a tendency to become more accurate with experience.

\section{EXPERIMENT 5B}

In Experiment 5B, we assessed whether participants' estimates of board widths would improve over time, without varying arousal as in Experiment 5A. Participants viewed two sets of neutral pictures and estimated the board widths after viewing each set. This allowed us to assess any changes in width estimates due to practice. If participants' estimates decreased after the second set of neutral pictures, it would suggest that those estimates were altered by experience. However, if participants' estimates did not change, it would suggest that the results of Experiment 5A were due to higher rates of arousal, and not to experience with estimating.

\section{Method}

Participants. Thirteen (5 female, 8 male) University of Utah students participated in the experiment for credit in a psychology course. All participants could maintain a normal posture and had normal or corrected-to-normal vision. All participants were naive as to the purpose of the experiment and gave written informed consent to participate.

Apparatus. All apparati were the same as those used in Experiments 1,2,3, and 5A, except for the addition of pictures selected from Lang et al.'s (1999) IAPS. One hundred twenty images were selected and divided into two groups (first neutral and second neutral) of 60 pictures. ${ }^{3}$ Arousal was rated no differently between the first neutral set $(M=4.2, S E=0.09)$ and the second neutral set $(M=4.0, S E=0.18)$ of pictures $[t(118)=1.32, p=.19]$. Valence was rated no differently between the first neutral set $(M=$ $5.3, S E=0.26)$ and the second neutral set $(M=5.6, S E=1.3)$ of pictures $[t(119)=-0.93, p=.35]$. Images were presented using PowerPoint.

Design. The design was identical to that in Experiment 5A, but there was no manipulation of the content of the images. All presented images were neutral.

Procedure. The procedure was identical to that in Experiment $5 \mathrm{~A}$. However, the arousing set of pictures was replaced with a second set of neutral pictures.

\section{Results and Discussion}

Response bias check. As in Experiment 5A, none of the participants were able to verbalize the true hypothesis of the experiment. Most participants made statements such as "... to see how accurate people are at estimating widths" or ". . . to see what I thought was interesting about the pictures." We are confident that participants believed that they participated in two separate experiments and did not alter or bias their estimates due to their thoughts about the nature of the experiment.

Arousal ratings. A paired-samples $t$ test was used to compare participants' ratings of their level of arousal when they were viewing each set of pictures. Analyses revealed that participants' level of arousal was no different when viewing the first set of neutral pictures than when viewing the second $[t(12)=-0.76, p=.46]$.

Width estimates. A 2 (condition: first neutral, second neutral) $\times 2$ (block: first, second) $\times 4$ (width: $1.75,3.65$, $8.73,13.5 \mathrm{~cm})$ repeated measures ANOVA, with all factors as within participants, revealed no significant main effect of condition $[F(1,12)=0.09, p=.77]$. Participants' estimates the first time they viewed the boards' widths $(M=8.88, S E=0.14)$ were no different from the second time they viewed the boards' widths $(M=8.96, S E=$ $0.14)$. There was no main effect of block $[F(1,12)=2.75$, $p=.12]$. There was a significant main effect of width $\left[F(3,36)=283.4, M S_{\mathrm{e}}=1.08, p<.001, \eta_{\mathrm{p}}^{2}=.96\right]$.

Precision of estimates. From a motivation similar to that in Experiments 1, 3, 4, and 5A, we analyzed whether viewing images influenced the precision with which participants estimated the board widths. Error terms were created as in Experiments 1, 3, 4, and 5A. The same repeated measures ANOVA used to evaluate changes in mean es- 
timations of width was used to evaluate the error terms. There was no significant effect of condition $[F(1,12)=$ $0.65, p=.44]$, block $[F(1,12)=0.40, p=.54]$, or width $[F(3,36)=1.99, p=.13]$. The null result of condition suggests that participants did not become more precise the second time they viewed the boards.

Participants' estimates of board width did not change after viewing the second set of neutral pictures from estimates made after viewing the first set of neutral pictures. This suggests that participants did become more accurate in estimating board width over time. Furthermore, Experiment 5B suggests that the results found in Experiment 5A were most likely due to the manipulation of arousal, rather than to changes in accuracy over time.

\section{GENERAL DISCUSSION}

In a series of experiments, we found that balance, through arousal, influenced size perception. Specifically, we showed that when participants were in a state of physical imbalance, they judged the width of a beam to be significantly smaller than when in a state of physical balance. Furthermore, we believe that this relationship may be due to the heightened level of arousal that can be experienced when in a state of physical imbalance.

In Experiment 1, we manipulated balance and recorded participants' size-matched judgments of beam width. Results indicated that participants estimated the width of the beam to be significantly smaller when they were unbalanced than when balanced. Experiments 2, 3, 4, and 5 isolated aspects of being unbalanced that could have contributed to the findings of Experiment 1. Specifically, Experiment 2 indicated that the amount of time that participants viewed the beam was not a contributing factor to the perceptual reduction of space. In Experiment 3, results suggested that higher states of arousal and gross motor movements, experienced both when jogging and when unbalanced, are sufficient to influence the perception of spatial layout. In Experiment 4, we found that higher levels of arousal produced without movements of the body could also influence the perception of spatial layout. In all previous conditions that produced an effect (unbalanced, jogging, and counting), participants not only were aroused, but also performed two tasks simultaneously. Experiments $5 \mathrm{~A}$ and $5 \mathrm{~B}$ showed that higher levels of arousal, not produced when participants performed a concurrent secondary task or experienced gross movements, were sufficient to alter estimates of the boards' widths. Collectively, gross body movements and dual tasks are not necessary to influence size judgments; however, heightened levels of arousal (caused by imbalance, jogging, counting, or viewing arousing images) are sufficient to influence size estimates.

These experiments add to the literature investigating the relationship between balance and visual perception. Previous research has demonstrated multiple instances in which visual information was necessary for the control of balance (Edwards, 1946; Paulus et al., 1984) and that when visual information was systematically manipulated, it had direct consequences for postural sway (Bertenthal \& Bai, 1989; Stoffregen et al., 1987). The present set of experiments extends these findings by demonstrating that balance can also influence visual perception. Specifically, we showed that when participants were unbalanced, they estimated the beam to be significantly smaller than when they were balanced. This effect seems to have been largely driven by the level of arousal that was experienced when the participants were unbalanced, jogging, counting, or viewing images.

The present work also adds to the literature that has documented a relationship between arousal and balance (Ohno, Wada, Saitoh, Sunaga, \& Nagai, 2004). Ohno et al. measured participants' postural sway with eyes open and closed at two time points. In addition, the authors recorded participants' state-level anxiety at each time point. They found that levels of anxiety and postural control positively correlated. Specifically, when participants' eyes were open, increases in level of anxiety were associated with more sway. However, they found no effect when participants closed their eyes. The authors speculated that higher levels of anxiety served to influence the processing of visual stimuli and, consequently, altered postural sway. The experiments presented in this article support Ohno et al.'s speculation that higher levels of arousal influence visual perception.

The mechanism by which arousal influences perception, especially size perception, is currently unknown. However, we will speculate about a few potential mechanisms. Stefanucci and Storbeck (2009) found that participants who viewed arousing images (that were unrelated to heights) subsequently judged heights to be greater than did participants who viewed neutral images. They suggested that when an observer views a height from above, cues to distance that may be normally used to judge extents are reduced (no texture gradient, no eye height information, etc.). If the observer happens to be more aroused, he or she may use that arousal as a cue, given a lack of other distal cues. Previous work by Zillman (1971) also suggests that arousal may be used as a cue, regardless of its source, when one is faced with uncertain information. Interestingly, Stefanucci and Storbeck found that heightened levels of arousal did not influence estimates of horizontal distances, only estimates of heights. They concluded that arousal may serve as a cue for estimating distance only when cues to distance are reduced (as on a height) and when the extent itself is potentially dangerous (as in the case of heights, but not horizontal distances). Cues to the width of the boards were likely available in these experiments, but the boards were raised off the ground, creating a potentially risky situation for action. This danger could have been perceived as greater when one considers the relatively small widths of the boards and, in some experiments, the fact that participants were unbalanced. When faced with a potentially risky situation, participants in our study may have relied on their heightened arousal as a cue for judging the widths of the boards. We speculate that, even with full cues, a risky situation may prompt the use of arousal as a cue for width estimates.

Similarly, Proffitt (2006) suggested that perception is the product of the optical information present in the environment and the associated costs of acting in the environment. In the present experiments, the perceived costs of walking across the beam may have been increased 
when participants were aroused or unbalanced. We believe that increasing the instability of participants raised the perceived associated costs of interacting with the environment, and arousal may have signaled this cost. For instance, greater instability may signal a higher chance of falling if one were to attempt to walk across a narrow beam. The reduced perceived width of the board as the result of physical imbalance, and by way of an increased state of arousal, may serve to dissuade action on the board. In this manner, the reduced perceived width of the board serves as an adaptive warning to the organism to be more cautious or to find an alternate route.

There is yet another, and possibly more direct, mechanism by which arousal could alter size perception. Previous work has demonstrated that level of arousal is associated with pupil dilation. Specifically, researchers have found that heightened states of arousal caused by loud noises (above 90 dB; Nunnally, Knott, Duchnowski, \& Parker, 1967; Patrick, 1969), lifting weights (10-30 lbs; Nunnally et al., 1967; Parker \& Mogyorosy, 1967), viewing emotionally laden images (Bradley, Miccoli, Escrig, \& Lang, 2008; Sturgeon, 1968), and smelling adverse smells (Kirby, 1968 ) all dilate the pupil. When illumination is controlled, greater pupil dilation is associated with a shorter depth of field and a blurred image (Bruce, Green, \& Georgeson, 2003). A shorter depth of field could cue the observer that the viewed object is closer and, therefore, smaller. It is possible that when aroused in our experiments, participants' pupils dilated and their perception of the width of the board changed as a direct consequence of this dilation.

Furthermore, if arousal influences size perception, as we have found here, it is worthy to discuss which levels of arousal are necessary to produce a change in perceptual estimates. In the imbalanced condition, participants experienced high levels of sway and, presumably, higher levels of arousal than in the balanced condition. However, we do not know how this level of arousal compared with that evoked by the counting task. Future studies should manipulate the intensity of arousal and record the level of arousal more precisely over time. Previous work has shown that the relationship between arousal and pupil dilation is nonlinear (Patrick, 1969). Specifically, it was found that pupil dilation occurred only after a certain level of arousal was reached. If pupil dilation is the mechanism by which perception of size is altered, we would expect that size perception would be affected only by those manipulations that reach a certain threshold of arousal. In future studies, it would be useful and important to measure levels of arousal over the course of the experiment in order to correlate possible peaks in arousal with specific perceptual estimates.

In addition, it would be interesting to document the degree of sway needed to influence width estimates. Due to the design of the balance board used in the present experiments, participants experienced high levels of sway. Future studies could manipulate sway to a lesser degree and assess the relative influence on width estimates. One possibility is that width estimates would decrease as sway increased. Alternatively, board width estimates may not be influenced until the degree of sway reaches a "dangerous" or arousing level.
One possible concern is that the observed results were due to methodological issues. Specifically, when using the tape measure for the size-matching task, the experimenter always started with the tape measure outstretched and the participants moved it in (to be more narrow). If participants were distracted from the size-matching task by the secondary task (unbalanced and jogging), the tape measure could have been narrowed more because participants were distracted. However, this argument does not explain the significant results of Experiment 4. In Experiment 4, the size-matching task was completed using a laptop display. The distance between the two lines depicted in the display started at a random distance from each other on every trial. Participants moved the poles in the display closer to or farther from one another. If the results of the experiments that did not use this display were due to distraction alone, we would expect null results in Experiment 4. This was not the case.

Previous work has raised concerns that a change in a perceptual measure does not necessarily equate to a change in the underlying perception; rather, the change could reflect a response bias that occurs after perceptual processes (Durgin et al., 2009; Russell \& Durgin, 2008). Demand characteristics, or how the participant believes he or she should respond given the manipulation, could also influence participants' responses to the task. However, two pieces of evidence suggest that these concerns may be allayed in our experiments. First, if participants guessed the hypothesis and made judgments accordingly, we would expect an order $\times$ condition interaction in our experiments. If demand characteristics were present, then the first time that participants estimated the board width, there should have been no difference between conditions, because they were unaware of the manipulation at that point and may have been unlikely to adjust their estimates. Once the manipulation was revealed, participants in the neutral-then-arousal order should have estimated the widths as smaller the second time they judged them. Conversely, participants in the arousal-then-neutral order should have estimated the widths as wider the second time they were judged. This would have resulted in a large effect of condition, which was completely driven by the second time participants estimated widths. However, there were no significant order $\times$ condition interactions for Experiments 1-4. Second, in Experiment 5, we asked participants to report what they thought to be the purpose of the experiment. None of the participants were able to correctly surmise the hypothesis. In addition, only 2 were able to suggest that the pictures were related to the estimations of width. However, neither of these participants was able to report a direction for that relationship. For these reasons, we are fairly confident that participants were not basing their estimates on what they believed to be the hypothesis of the experiment, although it is possible that these demands were present in a more unconscious manner.

It is also important to note that participants were instructed to imagine walking across the beam before each width estimate. Witt et al. (2005) found that there must be an intention to act in the environment for the environment 
to be perceptually rescaled with a manipulation to bodily state or physiological ability. In our experiments, being more aroused may have rescaled the perception of the beam's width only because participants were prompted to imagine walking across the beam. If we had not prompted participants to imagine walking across the beam, then we might not have found these effects. Future research should test whether priming an intention to act is necessary to produce effects of balance and arousal on perception.

However, this does not mean that the method by which one is aroused must also relate to the action. The counting task, a task unrelated to walking across the boards, did influence width estimations. We believe that higher states of arousal, regardless of why or how arousal was increased, may serve as a cue to visual size. Previous work by Zillman (1971) suggests that arousal is used as a cue when one is faced with uncertain information. In addition, previous experiments have shown that the counting task described in Experiment 4 increases physiological arousal (Budzynski \& Peffer, 1980; Harvey, 1980; S. W. Lee \& Guck, 1990; Maki \& McIlroy, 1996; Noteboom et al., 2001; Schneider, 1979; Yardley et al., 1995). Physiological arousal has also been shown to increase estimates of heights (Stefanucci \& Storbeck, 2009). In Stefanucci and Storbeck's experiments, participants' physiological arousal was increased by viewing pictures unrelated to heights. In our experiments, being unbalanced, jogging, counting, and viewing pictures all influenced size estimates, presumably by raising levels of arousal. The present set of experiments suggests that the method used to arouse may be irrelevant for physiological arousal's influence on estimates of spatial extents.

Another interesting idea would be to determine whether participants need to be aroused, when viewing the board, making the size estimate, or both, to see changes in perception. Specifically, manipulating the point during the procedure at which the participant is aroused would answer a few interesting questions. For example, determining whether being aroused when making size judgments is necessary to change those perceptual estimates could have implications for the lasting effects of these changes on perception. It could be that participants, if aroused when viewing the beam, encode the width of the beam as being smaller. If an individual always viewed a narrow board when aroused, he or she might refrain from walking across the beam more than would an individual who has viewed the board only when not aroused. In other words, an individual's experiences with the beam could influence later judgments and interactions with the beam even when arousal is not evoked. That is to say, the state of the body during individual experiences in interacting with the environment may shape not only the current judgments of that environment but also future interactions.

Research similar to the present experiments should also include populations who sway more or less, such as older populations. Older adults tend to be less stable and experience a greater number of falls than do younger individuals (Greenhouse, 1994; Sattin, 1992; Tibbits, 1996). It would be interesting to see whether older populations judge the width of the beams or other surfaces to be smaller than younger adults do, given the former's greater likelihood of falling. Furthermore, research that investigates changes to perception in older adults could help develop training programs for improving balance and understanding the implications of being unbalanced (including perceptual implications) to reduce instances of falls.

\section{Conclusions}

Previous research has demonstrated that manipulating visual information affects balance. In a series of experiments, we demonstrated that arousal, sometimes produced by physical imbalance, influenced the perception of beam width. Across four experiments, participants who were aroused due to being imbalanced, jogging, counting, or viewing images estimated the width of beams to be significantly smaller than when not aroused. We conclude that a high level of arousal, without gross bodily movement or increased cognitive load, is sufficient to influence size perception. This work contributes to the literature that has investigated the relationship between vision, balance, and arousal.

\section{AUTHOR NOTE}

This research was supported in part by NSF Grant IIS-0914488, for which the second author is a co-PI, and by NIH Grant RO1MH07578101A2, for which the second author serves as a consultant. The authors thank Rebecca Koppel, Alex Leal, and Jose Dominguez for their help in collecting the data. Correspondence concerning this article should be addressed to M. N. Geuss, Department of Psychology, University of Utah, 380 S 1530 E, Room 502, Salt Lake City, UT 84112 (e-mail: michaelgeuss@gmail.com).

\section{REFERENCES}

Andersen, G. J., \& Dyre, B. P. (1989). Spatial orientation from optic flow in the central visual field. Perception \& Psychophysics, 45, 453458.

Bardy, B. G., Warren, W. H., \& Kay, B. (1999). The role of central and peripheral vision in postural control during walking. Perception \& Psychophysics, 61, 1356-1368.

Bertenthal, B. I., \& BAI, D. L. (1989). Infant sensitivity to optical flow for controlling posture. Developmental Psychology, 25, 936-949.

Bertenthal, B. I., Rose, J. L., \& Bai, D. L. (1997). Perception-action coupling in the development of visual control of posture. Journal of Experimental Psychology: Human Perception \& Performance, 23, 1631-1643.

Bradley, M. M., Codispoti, M., Cuthbert, B. N., \& Lang, P. J. (2001). Emotion and motivation: I. Defensive and appetitive reactions in picture processing. Emotion, 1, 276-298.

BradLEY, M. M., \& LANG, P. (2007). The International Affective Picture System (IAPS) in the study of emotion and attention. In J. A. Coan \& J. J. B. Allen (Eds.), Handbook of emotion elicitation and assessment (pp. 29-46). Oxford: Oxford University Press.

Bradley, M. M., Miccoli, L., Escrig, M. A., \& LANG, P. J. (2008). The pupil as a measure of emotional arousal and autonomic activation. Psychophysiology, 45, 602-607.

Bronstein, A. M., \& Buckwell, D. (1997). Automatic control of postural sway by visual motion parallax. Experimental Brain Research, 113, 243-248.

Bruce, V., Green, P. R., \& Georgeson, M. A. (2003). Visual perception: Physiology, psychology, and ecology. New York: Psychology Press.

Budzynski, T. H., \& Peffer, K. E. (1980). Biofeedback training. In I. L. Katash \& L. B. Schlesinger (Eds.), Handbook on stress and anxiety (pp. 413-427). San Francisco: Jossey Bass.

Butterworth, G., \& Hicks, L. (1977). Visual proprioception and postural stability in infancy: A developmental study. Perception, 6, $255-262$. 
Dijkstra, T. M., Schoner, G., Giese, M. A., \& Gielen, C. C. (1994). Frequency dependence of the action-perception cycle for postural control in a moving visual environment: Relative phase dynamics, Biological Cybernetics, 71, 489-501.

Durgin, F. H., Baird, J. A., Greenburg, M., Russell, R., ShaughNessy, K., \& Waymouth, S. (2009). Who is being deceived? The experimental demands of wearing a backpack. Psychonomic Bulletin \& Review, 16, 964-969.

EDWARDS, A. S. (1946). Body sway and vision. Journal of Experimental Psychology, 36, 526-535.

Greenhouse, A. H. (1994). Falls among the elderly. In M. L. Albert \& J. E. Knoefel (Eds.), Clinical neurology of aging (2nd ed., pp. 611626). New York: Oxford University Press.

Harvey, L. (1980). Patterns of response to stressful tasks. Research Bulletin of the Himalayan International Institute, 2, 4-8.

HoRAK, F. B. (1987). Clinical measurement of postural control in adults. Physical Therapy, 67, 1881-1885.

Kapoula, Z., \& ThanH-Thuan, L. (2006). Effects of distance and gaze position on postural stability in young and old subjects. Experimental Brain Research, 173, 438-445.

Kerr, B., Condon, S. M., \& McDonald, L. A. (1985). Cognitive spatial processing and the regulation of posture. Journal of Experimental Psychology: Human Perception \& Performance, 11, 617-622.

KIRBY, M. W. (1968). The pupil reaction in response to an unpleasant odor as an index of affect. Unpublished master's thesis, Wake Forest University, Winston-Salem, NC.

Kunkel, M., Freudenthaler, N., Steinhoff, B. J., Baudewig, J., \& Paulus, W. (1998). Spatial-frequency-related efficacy of visual stabilization of posture. Experimental Brain Research, 121, 471-477.

Lajoie, Y., Teasdale, N., Bard, C., \& Fleury, M. (1993). Upright standing and gait: Are there changes in attentional requirements related to normal aging? Experimental Aging Research, 22, 185-198.

LANG, P., Bradley, M., \& CuthBert, B. (1999). International Affective Picture System (IAPS): Technical manual and affective ratings. Gainsville: University of Florida, Center for Research in Psychophysiology.

LeE, D. N., \& Aronson, E. (1974). Visual proprioceptive control of standing in human infants. Perception \& Psychophysics, 15, 529532.

LeE, D. N., \& Lishman, J. R. (1975). Visual proprioceptive control of stance. Journal of Human Movement Studies, 1, 87-95.

LEE, S. W., \& GucK, T. P. (1990). The use of mental arithmetic (serial 7's) as a stressor in psychophysiological stress profiling: A preliminary report. Medical Psychotherapy, 3, 97-102.

LORD, S. R., \& WARD, J. A. (1994). Age-associated differences in sensori-motor function and balance in community dwelling women. Age \& Ageing, 23, 452-460.

MaKi, B. E., \& McIlroy, W. E. (1996). Influence of arousal and attention on the control of postural sway. Journal of Vestibular Research: Equilibrium \& Orientation, 6, 53-59.

Mark, L. S., Balliet, J. A., Graver, K. D., Douglas, S. D., \& Fox, T. (1990). What an actor must do in order to perceive the affordance for sitting. Ecological Psychology, 2, 325-366.

Noteboom, T. J., Fleshner, M., \& ENOKA, R. M. (2001). Activation of the arousal response can impair performance on a simple motor task. Journal of Applied Physiology, 91, 821-831.

Nunnally, J. C., Knott, P. D., Duchnowski, A., \& Parker, R. (1967). Pupillary response as a general measure of activation. Perception \& Psychophysics, 2, 149-155.

Ohno, H., Wada, M., Saitoh, J., Sunaga, N., \& Nagai, M. (2004). The effect of anxiety on postural control in humans depends on visual information processing. Neuroscience Letters, 364, 37-39.

Parker, R. K., \& Mogyorosy, R. S. (1967, September). Pupillary response to induced muscular tension. Paper presented at the 75 th Annual Convention of the American Psychological Association, Washington DC.

PATRICK, M. S. (1969). Pupillometry as a method of assessing stress due to noise. Southhampton, U.K.: Institute of Sound and Vibration Research.

Paulus, W. M., Straube, A., \& Brandt, T. (1984). Visual stabilization of posture: Physiological stimulus characteristics and aspects. Brain, 107, 1143-1163.
ProffitT, D. R. (2006). Embodied perception and the economy of action. Perspectives on Psychological Science, 1, 1-13.

Riccio, G. E., \& Stoffregen, T. A. (1988). Affordances as constraints on the control of stance. Human Movement Science, 7, 265-300.

Russell, R., \& Durgin, F. H. (2008). Demand characteristics, not effort: The role of backpacks in judging distance [Abstract]. Journal of Vision, 8(6), 755a.

SATtin, R. W. (1992). Falls among older persons: A public health perspective. Annual Reviews of Public Health, 13, 489-508.

SchneIDER, C. (1979). Foundations of biofeedback practice: Workshop manual. Wheat Ridge, CO: Biofeedback Society of America.

Stefanucci, J. K., \& Storbeck, J. (2009). Don't look down: Emotional arousal elevates height perception. Journal of Experimental Psychology: General, 138, 131-145.

Stoffregen, T. A. (1985). Flow structure versus retinal location in optical control of stance. Journal of Experimental Psychology: Human Perception \& Performance, 11, 554-565.

Stoffregen, T. A., Schmuckler, M. A., \& Gibson, E. J. (1987). Use of central and peripheral optical flow in stance and locomotion in young walkers. Perception, 16, 113-119.

Stoffregen, T. A., Smart, L. J., Bardy, B. G., \& Pagulayan, R. J. (1999). Postural stabilization of looking. Journal of Experimental Psychology: Human Perception \& Performance, 25, 1641-1658.

Sturgeon, R. S. (1968). The effect of a color slide hierarchy for fear stimuli on the reduction of fear of snakes as measured by the pupillometric technique. Dissertation Abstracts, 29, 3222A-3223A.

Tibits, G. M. (1996). Patients who fall: How to predict and prevent injuries. Geriatrics, 51, 24-28.

Witt, J. K., Proffitt, D. R., \& Epstein, W. (2005). Tool use affects perceived distance, but only when you intend to use it. Journal of Experimental Psychology: Human Perception \& Performance, 31, 880-888.

Woollacott, M., \& Shumway-Cook, A. (2002). Attention and the control of posture and gait: A review of an emerging area of research. Gait \& Posture, 16, 1-14.

Wright, D. B. (2007). Graphing within-subjects confidence intervals using SPSS and S-Plus. Behavior Research Methods, 39, 82-85.

YARdley, L., Watson, S., Britton, J., Lear, S., \& Bird, J. (1995). Effects of anxiety arousal and mental stress on the vestibulo-ocular reflex. Acta Oto-Laryngologica, 115, 597-602.

Zillman, D. (1971). Excitation transfer in communication-mediated aggressive behavior. Journal of Experimental Social Psychology, 7 419-434.

\section{NOTES}

1. SEs were calculated using the method outlined in Wright (2007) for this graph and all other graphs.

2 . The photos associated with the following IAPS image numbers were used: $1030,1040,1051,1120,1220,1240,1300,1301,1302,1321$, $1540,1590,1600,1603,1604,1610,1930,1931,2040,2070,2100$ $2110,2372,2383,2800,2900,4599,4601,4606,4608,4653,4660$, $5000,5001,5010,5030,5201,5220,5470,5500,5510,5621,5628$, $5629,5660,5700,5731,5750,5800,5900,5950,5970,5982,5991$, $6230,3250,6260,6300,6510,6570,6900,6910,7000,7004,7009$, $7010,7025,7030,7034,7035,7040,7050,7080,7090,7095,7096$, $7100,7130,7140,7150,7175,7186,7187,7190,7205,7207,7211$, $7224,7237,7238,7490,7500,7545,7560,7595,7640,7705,7820$, $7830,8021,8030,8031,8040,8041,8080,8160,8161,8180,8185$, $8200,8250,8260,8300,8340,8350,8370,8400,8470,9622$.

3 . The first neutral set was the same set used in Experiment 5A. The photos associated with the following IAPS image numbers were used for the second neutral set: 1450,1670,1710,1931, 2058, 2190, 2221, 2276, $2320,2370,2381,2389,2391,2480,2620,2681,2850,2870,2880$, 2890, 5010, 5390, 5500, 5510, 5520, 5530, 5628, 5629, 5740, 5800, 5910, 5920, 7009, 7025, 7030, 7031, 7040, 7090, 7100, 7110, 7140, 7190, 7205, 7224, 7234, 7235, 7260, 7380, 7640, 8034, 8041, 8050, $8080,8090,8116,8170,8231,8500,8502,9611$.

(Manuscript received June 10, 2009 revision accepted for publication April 26, 2010.) 